\title{
Non-Isothermal Crystallization Kinetics of Green Microalgae Filled Poly(Lactic Acid)
}

\author{
M. Kodal*, T. Kutluk, T. Yarici, N. Kapucu and G. Ozkoc \\ Department of Chemical Engineering, Kocaeli University, 41380 Kocaeli, Turkey
}

(Received August 2, 2019; revised version September 9, 2019; in final form September 15, 2019)

\begin{abstract}
In this study, the effect of green microalgae as a nucleating agent on the non-isothermal crystallization of poly(lactic acid) (PLA) was investigated by differential scanning calorimeter and polarized optical microscope. The parameters of non-isothermal crystallization kinetics of PLA and PLA/green microalgae composites were obtained through common kinetic models such as Avrami, Ozawa, and combined Avrami and Ozawa. Moreover, nucleating activity of green microalgae and activation energies of composites were determined by the Dobreva-Gutzow and the Friedman methods, respectively. Dispersion of green microalgae in PLA was characterized using scanning electron microscopy. PLA/green microalgae composites having different green microalgae content from 5 to $20 \mathrm{wt} \%$ were prepared via solvent casting method. Although some agglomerated green microalgae was observed in the PLA matrix, it was found that the green microalgae were homogeneously distributed within the matrix at its lower loading levels. The Avrami and combined Avrami-Ozawa results showed that PLA/green microalgae composites have faster melt crystallization rates than that of pure PLA. Nucleating activity of green microalgae was proved by the Dobreva and Gutzow kinetic approach. Moreover, polarized optical microscope analysis revealed that crystallization onset temperature of PLA shifted to higher temperature upon cooling in the presence of green microalgae.
\end{abstract}

DOI: 10.12693/APhysPolA.136.974

PACS/topics: non-isothermal crystallization kinetics, poly(lactic acid), green microalgae

\section{Introduction}

In recent years, interest in biodegradable polymers has significantly increased due to the increased sensitivity to environmental pollution and rising oil prices. Among the biodegradable polymers, PLA synthesized from renewable resources is the most widely used biopolymer due to its high stiffness and strength, which are similar to polystyrene (PS) [1]. Nowadays, PLA is used in fields such as packaging, clothing, construction, fiber, and weaving. On the other hand, slow crystallization rate of PLA restricts its versatile applications. In the literature, some works have been done to improve the crystallization rate of PLA. One of the recommended methods is to change the injection molding parameters. Harris and Lee [2] optimized the injection molding processing parameters to increase the final crystallinity of PLA. They concluded that alteration of process parameters resulted in a significant effect on the final crystallinity of molded PLA. Another most widely used method to increase the crystallization rate of PLA is the incorporation of nucleating agents to the PLA. There are many nucleating agents used in the literature for this purpose. Liao et al. [3] investigated the nucleating agent efficiency of $\mathrm{CaCO}_{3}, \mathrm{TiO}_{2}$, and $\mathrm{BaSO}_{4}$ on isothermal cold crystallization of PLA. They found that crystallization rate of PLA decreased with increasing crystallization temperature in the presence of nucleating agents. In another study, it was stated that talc and $\mathrm{CaCO}_{3}$

*corresponding author; e-mail: mehmet.kodal@kocaeli.edu.tr increased the degree of crystallinity of PLA [4]. In a recent research, a nucleating agent, aromatic sulfonate derivative (LAK301), on thermal behaviors of PLA was evaluated by differential scanning calorimeter (DSC). The authors found that LAK301 acted as a very efficient nucleating agent by increasing crystallinity percentage from $5.6 \%$ in pure PLA [5]. Amino acids and poly(amino acids) were used as nucleating agents for PLA by Carbone et al. in 2015 [6]. They indicated that polyglycine possessed a significant nucleating ability. The effect of talc on the crystallinity of PLA was investigated by Shakoor and Thomas [7]. They showed that the crystallinity of PLA increased from $2 \%$ to $25 \%$ in the presence of talc. In our previous studies, we used novel nano-fillers (POSSs) to improve the crystallization rate of PLA. We found that POSSs nanoparticles acted as nucleating agents and crystallization stage began at higher temperatures upon cooling $[8,9]$.

Recently, marine algae were suggested as potential fillers or reinforcing agent in composites to control their proliferation occurred in coastlines due to environmental problems [10]. Chiellini et al. used green algae as a filler for poly(vinyl alcohol) (PVA). They found that Young's modulus of PVA sharply increased by increasing the algae percentage in the composites [11]. The same authors used sea-weed Zostera marina as a possible filler in eco-compatible hybrid composites. They stated that decreasing Zostera fibers size from $3 \mathrm{~m}$ to $2 \mathrm{~mm}$ produced hybrid molecules more flexible (higher elongation, lower tensile strength and modulus) [12]. Iannace et al. prepared by mixing thermoplastic biodegradable polymers with sea algae fibers. They found that composites showed higher elastic modulus and lower strength than the matrix components [13]. Morphology and thermal 
mechanical properties of poly ( $\varepsilon$-caprolactone) (PCL) and poly(hydroxyl butyrate) (PHB) blends including seaweed fibers were investigated by Bargnini et al. They found that addition of algal fibers improved the Young modulus of the blends, creating a concomitant loss in elongation at break and ultimate strength [14]. Buluta and Budtova used various algae (red, brown, and green) as filler to prepare PLA based composites via melt blending method. The Young modulus of composites increased at $40 \mathrm{wt} \%$ load of algae as compared with neat PLA although the strain at break and tensile strength decreased [10].

In addition to marine based microalgae, industrial large-scale production of microalgal biomass can be also used as a filler for polymers. It usually uses continuous culture during daylight. In this operation, fresh culture medium is fed at a constant rate and the same quantity of microalgal broth is introvert perpetually. In addition, methods of large-scale production of microalgae are raceway ponds and tubular photobioreactors [15]. The oil used as the biodiesel raw material is extracted from the dry microalgae and then the microalgae is formed as powder in large quantities afterwards. These wastes are environmentally friendly and they can be evaluated as additives to improve the crystallization behavior of polymers such as PLA.

In the current study, for the first time in the literature, it was aimed to investigate the non-isothermal crystallization behavior of PLA/green microalgae composites via differential scanning calorimeter (DSC) by applying different crystallization kinetics models. The crystallization morphology of PLA/green microalgae composite was analyzed by polarized optical microscopy (POM). Moreover, the dispersion of green microalgaes in PLA matrix was judged by scanning electron microscopy (SEM).

\section{Experimental}

\subsection{Materials}

PLA (PLI005) was purchased from NaturePlast Company, France. Microalgae strain (Chlorella variabilis) was obtained from Dr. Turgay Çakmak (Medeniyet University, Turkey).

\subsection{Cultivation of microalgae biomass}

Chlorella variabilis was cultivated in $8 \mathrm{~L}$ photo bioreactor and air was supplied to the reactor at a rate of $50 \mathrm{~L} / \mathrm{h}$. Illumination was provided by a four- $7 \mathrm{~W} 2700 \mathrm{~K}$ LED lamps. Then, cells were harvested by centrifugation and then microalgae paste was dried in an oven at $70^{\circ} \mathrm{C}$ for $12 \mathrm{~h}$.

\subsection{Preparation of films}

Before preparation of PLA based composites, PLA was dried in an oven at $50^{\circ} \mathrm{C}$ for $12 \mathrm{~h}$ under vacuum. PLA mixtures having different green microalgae loading levels $(5,10,15,20 \mathrm{wt} \%)$ were solubilized in chloroform. After that, the solutions were cast on glass Petri dishes, and the solvent was allowed to evaporate at room temperature. Finally, all the films were further dried overnight under vacuum at $50^{\circ} \mathrm{C}$ to eliminate the residual solvent.

\subsection{Characterization}

Morphological studies were performed with a scanning electron microscope (SEM, JEOL JSM 6060). The samples were cryofractured by hand after immersion in liquid nitrogen. The samples were gold coated before observations to prevent arching.

Differential scanning calorimeter (DSC) analysis was performed to investigate the non-isothermal crystallization kinetics of pure PLA, and PLA/green microalgae composites. Each DSC experiment was consisting of two segments. In the first segment, films were heated from 25 to $200^{\circ} \mathrm{C}$ with a heating rate of $15^{\circ} \mathrm{C} / \mathrm{min}$. They were kept at this temperature for 5 min to eliminate the thermal history. Then in the second step, they were cooled to $25^{\circ} \mathrm{C}$ at cooling rates of $1,2,5$, and $10^{\circ} \mathrm{C} / \mathrm{min}$. The kinetic data were produced by manipulating the cooling thermograms obtained in the second segment.

A polarizing optical microscope (Nikon LV100 POL) equipped with a Linkam THMSE 600 hot stage was used to observe the crystal nucleation and crystal growth of the PLA and PLA/green microalgae composites. The samples were first placed between two glass slides and then they were heated from 25 to $190^{\circ} \mathrm{C}$. In order to erase the thermal history, they were kept at this temperature for $10 \mathrm{~min}$, and then they were cooled to $90^{\circ} \mathrm{C}$ at a cooling rate of $1^{\circ} \mathrm{C} / \mathrm{min}$.

\section{Results and discussion}

\subsection{Scanning electron microscopy analysis}

The SEM micrographs of 5 and 20 wt\% green microalgae filled PLA composites are shown in Fig. 1. As can be seen the green microalgae particles dispersed homogeneously in PLA matrix at both loading levels. The average particle diameter of green microalgae in PLA matrix was in the range of 0.1 to $0.5 \mu \mathrm{m}$ for its lower loading level (5 wt\%). Moreover, some agglomerated particles were seen in PLA matrix. On the other hand, increasing algae content from $5 \mathrm{wt} \%$ to $20 \mathrm{wt} \%$ promoted agglomerations of green microalgae particles. Although dispersion of the green microalgae particles was well in the matrix, some agglomerates with the diameter of 8-12 $\mu \mathrm{m}$ were obtained for its higher loading levels.

\subsection{Non-isothermal crystallization kinetics}

A representative DSC thermograms obtained in cooling step of DSC experiments is given in Fig. 2. As can be seen, PLA did not exhibit any crystallization exotherm at a cooling rate of $10^{\circ} \mathrm{C} / \mathrm{min}$. On the contrary, the crystallization exotherms were obtained during cooling in the presence of green microalgae. Moreover, the crystallization peak become wider and slightly shifted 


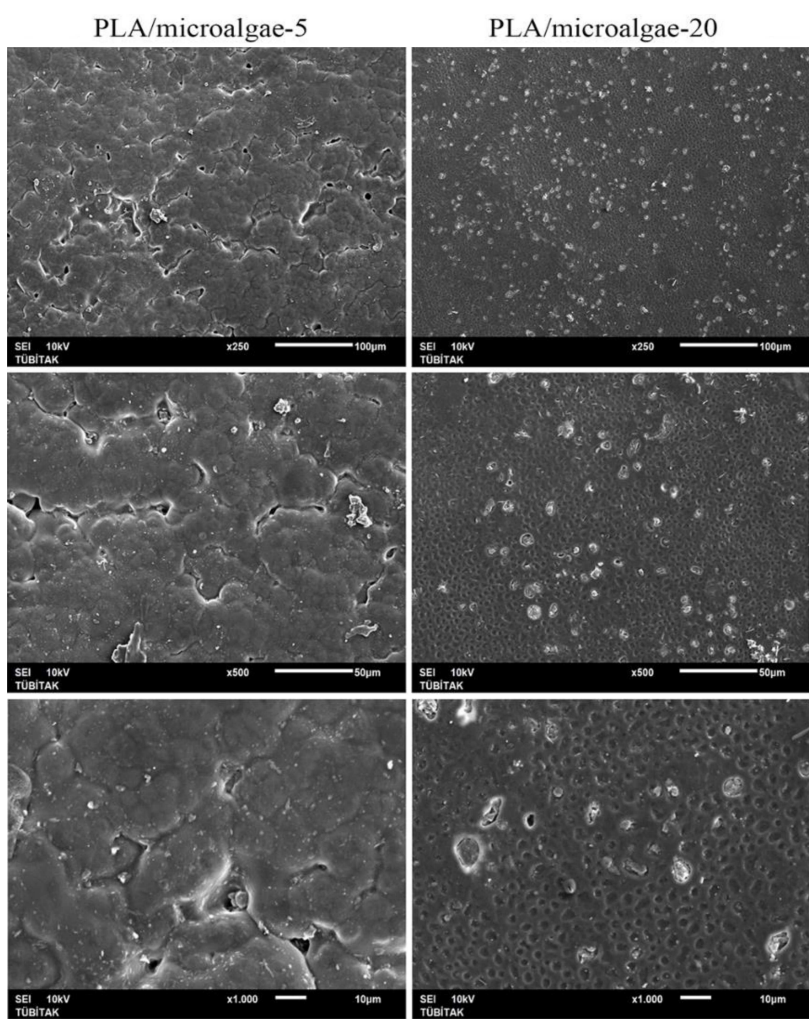

Fig. 1. SEM micrographs of PLA/algae composites with different magnifications $(250 \times, 500 \times$ and $1000 \times)$.

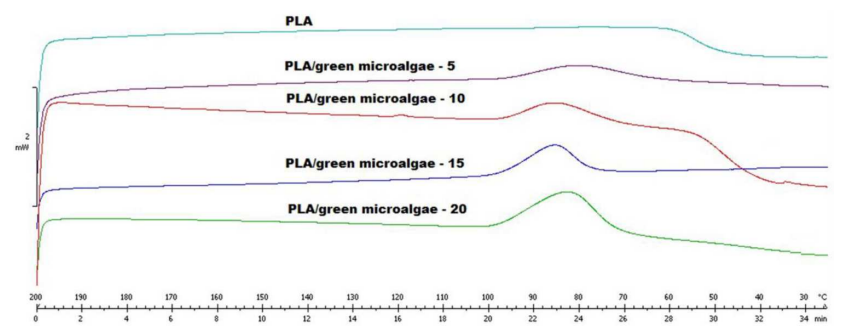

Fig. 2. Representative DSC thermograms of PLA and PLA/green microalgae composites (cooling rate: $10^{\circ} \mathrm{C} / \mathrm{min}$ ). Difference between $\mathrm{PLA} /$ green -5 and $\mathrm{PLA} /$ green -15 is around $2 \mathrm{~mW}$, what corresponds to the heat flux during DSC experiments.

to higher temperatures as green microalgae content increased in the composites.

Table I shows the crystallization onset and crystallization peak temperature and crystallization half-time of PLA and PLA/green microalgae composites obtained from the cooling segment. As can be seen from Table I, crystallization onset temperature of PLA significantly shifted to higher values in the presence of green microalgae at any given cooling rates. Moreover, it was found that all PLA/green microalgae composites exhibited crystallization behavior at higher cooling rates $\left(5^{\circ} \mathrm{C} / \mathrm{min}\right.$ and $\left.10^{\circ} \mathrm{C} / \mathrm{min}\right)$ as compared to pure PLA. This can be attributed to the nucleating activity of green microalgae.
TABLE I

Crystallization onset and crystallization peak temperature and crystallization half time of PLA and PLA/green microalgae composites obtained from the cooling segment.

\begin{tabular}{|c|c|c|c|c|}
\hline Sample & $\begin{array}{c}\text { Cooling } \\
\text { rate } \beta \\
{\left[{ }^{\circ} \mathrm{C} / \mathrm{min}\right]}\end{array}$ & $\begin{array}{c}T_{c . \text { onset }} \\
{\left[{ }^{\circ} \mathrm{C}\right]}\end{array}$ & $\begin{array}{c}T_{\text {c.endset }} \\
{\left[{ }^{\circ} \mathrm{C}\right]}\end{array}$ & $\begin{array}{l}\text { Crystal. } \\
\text { half time } \\
t_{1 / 2}[\mathrm{~min}]\end{array}$ \\
\hline \multirow{4}{*}{ PLA } & 1 & 103.5 & 83.6 & 7.8 \\
\hline & 2 & 103.4 & 78.9 & 6.0 \\
\hline & 5 & - & - & - \\
\hline & 10 & - & - & - \\
\hline \multirow{4}{*}{$\begin{array}{l}\text { PLA/green } \\
\text { microalgae-5 }\end{array}$} & 1 & 116.6 & 85.0 & 15.4 \\
\hline & 2 & 110.1 & 84.3 & 6.9 \\
\hline & 5 & 104.6 & 72.1 & 4.1 \\
\hline & 10 & 94.6 & 64.6 & 1.2 \\
\hline \multirow{4}{*}{$\begin{array}{l}\text { PLA/green } \\
\text { microalgae-10 }\end{array}$} & 1 & 117.6 & 93.4 & 9.3 \\
\hline & 2 & 111.9 & 86.1 & 5.7 \\
\hline & 5 & 108.5 & 69.3 & 3.4 \\
\hline & 10 & 99.2 & 65.0 & 1.3 \\
\hline \multirow{4}{*}{$\begin{array}{l}\text { PLA/green } \\
\text { microalgae-15 }\end{array}$} & 1 & 118.4 & 83.7 & 15.6 \\
\hline & 2 & 113.1 & 81.8 & 5.4 \\
\hline & 5 & 109.7 & 71.4 & 4.5 \\
\hline & 10 & 101.9 & 60.4 & 1.1 \\
\hline \multirow{4}{*}{$\begin{array}{l}\mathrm{PLA} / \text { green } \\
\text { microalgae-20 }\end{array}$} & 1 & 119.6 & 88.1 & 14.3 \\
\hline & 2 & 116.1 & 86.2 & 5.0 \\
\hline & 5 & 111.5 & 70.6 & 4.4 \\
\hline & 10 & 100.1 & 65.0 & 1.8 \\
\hline
\end{tabular}

The relative crystallinity, $X(T)$, can be plotted as a function of temperature as follows:

$$
X(T)=\int_{T_{0}}^{T}\left(\frac{\mathrm{d} H_{c}}{\mathrm{~d} T}\right) \mathrm{d} T / \int_{T_{0}}^{T_{\infty}}\left(\frac{\mathrm{d} H_{c}}{\mathrm{~d} T}\right) \mathrm{d} T,
$$

where $T_{0}$ and $T_{\infty}$ are the onset and the endset temperatures of the crystallization process, respectively, $T$ is any temperature in crystallization process, and $\mathrm{d} H_{c}$ represents the differential crystallization enthalpy change in temperature range of $\mathrm{d} T$.

The relative crystallinity as a function of temperature for PLA and PLA/green microalgae composites is shown in Fig. 3.

All the curves have a sigmoidal shape. The nuclei are formed in the first plateau of the curves. At the descending linear second part of the curves, called as accelerated crystallization period, the crystallites begin to grow up. Finally, crystallization rate decreases as can be seen in the deviated part of the curves [16-18]. Moreover, the crystallization temperature decreased as the cooling rates increased. This situation resulted from the fact that time was enough for rearrangement of PLA chains at lower cooling rates $\left(1^{\circ} \mathrm{C} / \mathrm{min}\right.$ and $\left.2{ }^{\circ} \mathrm{C} / \mathrm{min}\right)[19,20]$.

For non-isothermal melt crystallization process, crystallization time $t$ can be obtained from Eq. (2):

$$
t=\left(T_{0}-T\right) / \beta,
$$


where $T_{0}$ is the initial crystallization temperature, $T$ is the temperature at the crystallization time of $t$, and $\beta$ is the cooling rate. Figure 4 shows the plots of relative crystallinity as a function of time for non-isothermal melt crystallization of pure PLA and green microalgae filled PLA composites.

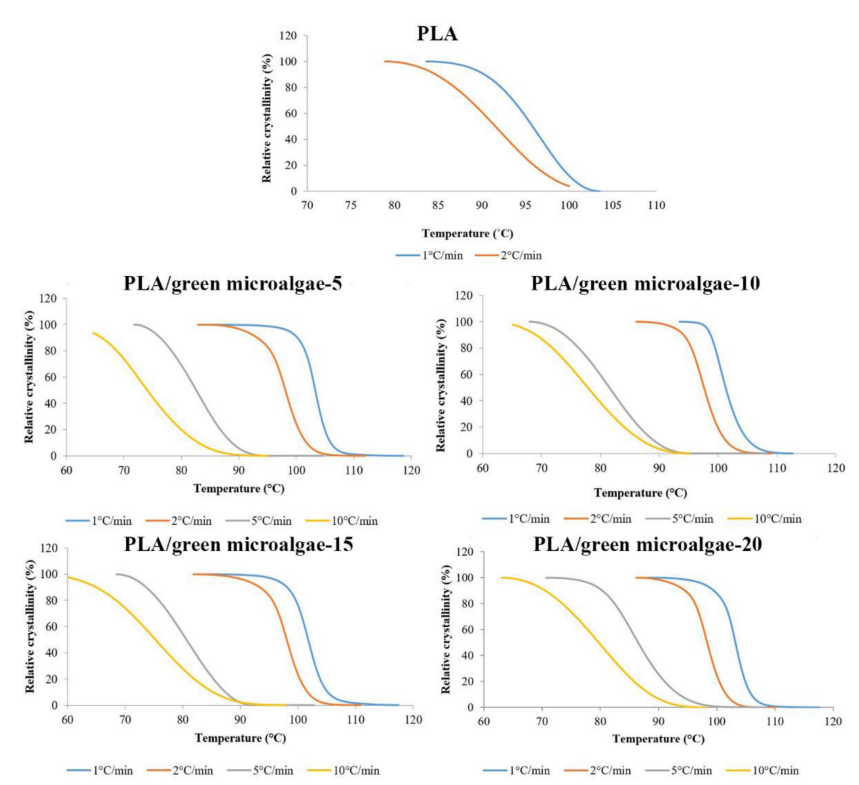

Fig. 3. Relative crystallinity of PLA and PLA/green microalgae composites as a function of temperature at different cooling rates.
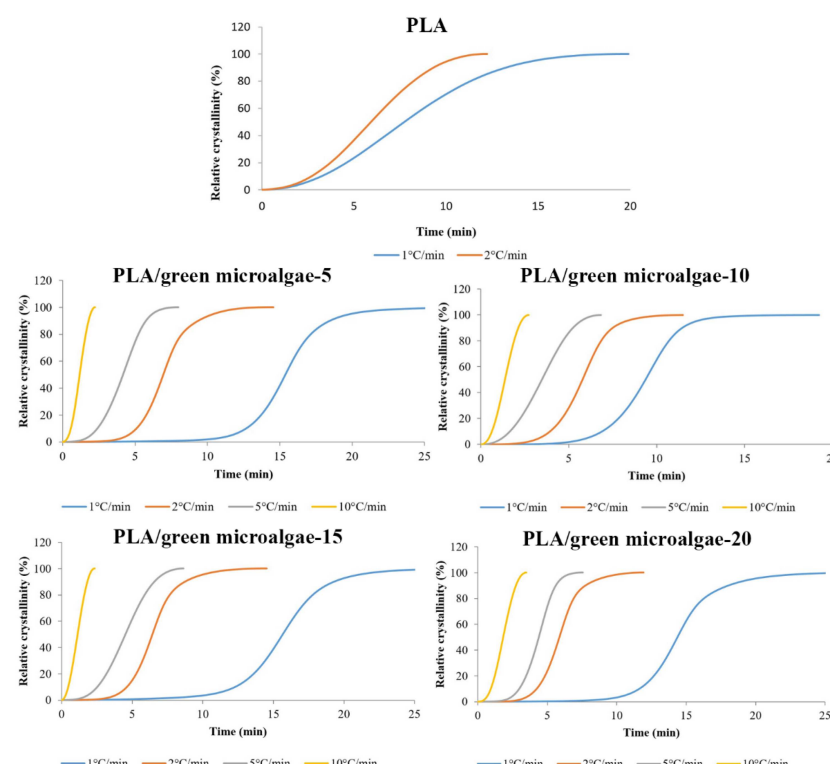

Fig. 4. Relative crystallinity of PLA and PLA/green microalgae composites as a function of time at different cooling rates.
Besides, the time required to complete $50 \%$ of the crystallization (the crystallization half time $t_{1 / 2}$ ), can be obtained from the curves of $X(t)$ vs. time [21]. Low $t_{1 / 2}$ values indicate higher crystallization rate. The crystallization half time $t_{1 / 2}$ ) values of pure PLA and PLA/green microalgae composites obtained from the curves at a given cooling rate were given in Table I. It is clear that the crystallization half time decreased as the heating rate increased for all composites. As can be seen from Table I, the $t_{1 / 2}$ values of PLA were higher in the presence of green microalgae at $1^{\circ} \mathrm{C} / \mathrm{min}$ cooling rate. On the other hand, crystallization rate of PLA increased and the time to reach the $50 \%$ relative crystallinity decreased at higher cooling rates in the presence of green microalgae. Regarding higher crystallization half time $\left(t_{1 / 2}\right)$ in the presence of green microalgae at the cooling rate of $1^{\circ} \mathrm{C} / \mathrm{min}$ might be attributed to the some degree of agglomeration of green microalgae in PLA matrix [22]. On the other hand, the $t_{1 / 2}$ value of PLA decreased in the presence of green microalgae for the other cooling rates, which showed that green microalgae enhanced the crystallization rate of PLA. It can be concluded that green microalgae advancing the melt crystallization significantly acted as a nucleating agent but extended the crystallization process of PLA.

The Avrami model and Eq. (3) are valid for isothermal crystallization $(T=$ const $)$, where the rate constant $Z_{t}$ is dependent on the crystallization temperature $T[23,24]$. $X(t)$ is the relative degree of crystallinity at time $t, Z_{t}$ is the rate constant that provides information about nucleation and growth rate, and the Avrami exponent $n$ gives information about nucleation type and morphology of the crystallite formed. The double logarithm of Eq. (3) is taken to calculate the rate constant $Z_{t}$ and the Avrami exponent $n$ (see Eq. (4)) [25]:

$$
\begin{aligned}
& 1-X(t)=\exp \left(-Z_{t} t^{n}\right), \\
& \ln (-\ln (1-X(t)))=\ln Z_{t}+n \ln t .
\end{aligned}
$$

It is well known that the equation is mainly used to describe isothermal conditions. It can only be used to explain the primary crystallization phase when transformed into non-isothermal conditions. As both nucleation and crystallization process are affected by continues change in temperature, the effectiveness of the crystallization rate should be taken into account $[19,26]$. Jeziorny stated that the Avrami model could be utilized for non-isothermal crystallization process, which is based on the assumption that the cooling rate remains constant for a given sample [25]. Jeziorny [27] revised the crystallization rate $Z_{t}$ in the Avrami equation dividing it by the cooling rate $\beta$ to incorporate the temperature change during the non-isothermal crystallization process as follows:

$$
\ln Z_{c}=\frac{\ln Z_{t}}{\beta} .
$$

The Avrami plots of $\ln (-\ln (1-X(t)))$ versus $\ln t$ for non-isothermal melt crystallization of pure PLA and PLA/green microalgae composites are shown in Fig. 5 


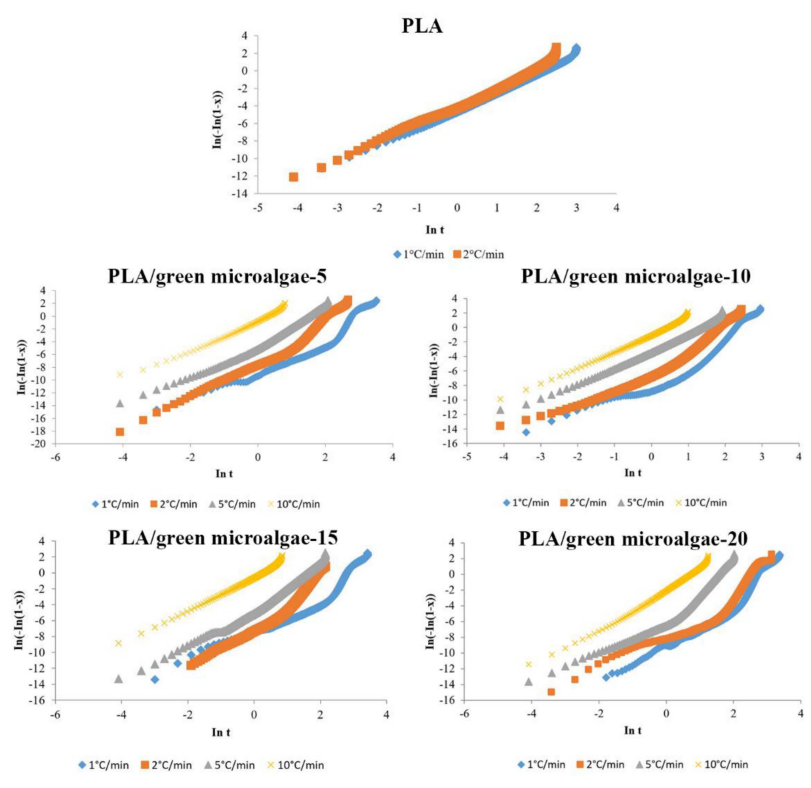

Fig. 5. Avrami curves of PLA and PLA/green microalgae composites.

TABLE II

Avrami kinetic parameters of PLA, and PLA/green microalgae composites at different cooling rates

\begin{tabular}{l|c|c|c|c|c}
\hline \hline \multicolumn{1}{c|}{ Sample } & $\begin{array}{c}\beta \\
{\left[{ }^{\circ} \mathrm{C} / \mathrm{min}\right]}\end{array}$ & $\mathrm{n}$ & $Z_{t}$ & $Z_{c}$ & $R^{2}$ \\
\hline \multirow{3}{*}{$\mathrm{PLA}$} & 1 & 2.11 & $4.61 \times 10^{-4}$ & $4.61 \times 10^{-4}$ & 0.996 \\
& 2 & 2.01 & $1.71 \times 10^{-3}$ & $4.13 \times 10^{-2}$ & 0.995 \\
& 5 & - & - & - & - \\
\hline & 10 & - & - & - & - \\
PLA/green & 1 & 2.35 & $6.29 \times 10^{-4}$ & $6.29 \times 10^{-4}$ & 0.992 \\
microalgae-5 & 5 & 2.20 & $1.80 \times 10^{-3}$ & $4.25 \times 10^{-2}$ & 0.998 \\
& 10 & 2.12 & $3.96 \times 10^{-3}$ & $3.30 \times 10^{-1}$ & 0.999 \\
& 1 & 2.30 & $3.60 \times 10^{-3}$ & $5.69 \times 10^{-1}$ & 0.997 \\
\hline & 2 & 2.25 & $1.88 \times 10^{-4}$ & $8.02 \times 10^{-4}$ & 0.996 \\
PLA/green & 5 & 2.15 & $3.71 \times 10^{-1}$ & $5.17 \times 10^{-1}$ & 0.999 \\
microalgae-10 & 10 & 2.13 & $3.74 \times 10^{-1}$ & $9.06 \times 10^{-1}$ & 0.995 \\
\hline & 1 & 2.60 & $4.06 \times 10^{-3}$ & $4.06 \times 10^{-3}$ & 0.997 \\
PLA/green & 2 & 2.21 & $6.14 \times 10^{-4}$ & $4.47 \times 10^{-2}$ & 0.998 \\
microalgae-15 & 5 & 2.14 & $6.50 \times 10^{-2}$ & $5.79 \times 10^{-1}$ & 0.995 \\
& 10 & 2.14 & $6.03 \times 10^{-1}$ & $9.50 \times 10^{-1}$ & 0.990 \\
\hline & 1 & 2.43 & $4.27 \times 10^{-3}$ & $4.27 \times 10^{-3}$ & 0.982 \\
PLA/green & 2 & 1.98 & $2.83 \times 10^{-3}$ & $5.32 \times 10^{-2}$ & 0.986 \\
microalgae-20 & 5 & 1.93 & $1.42 \times 10^{-1}$ & $6.77 \times 10^{-1}$ & 0.994 \\
& 10 & 1.70 & $8.51 \times 10^{-1}$ & $9.84 \times 10^{-1}$ & 0.991
\end{tabular}

and the parameters (the Avrami and Jeziorny constants) are listed in Table II. Each curve has a linear part in the beginning and inflection part indicating the spherulite impingement in the later stage [28]. These parts refer to the primary and secondary crystallization, respectively. Nucleation and subsequent crystal growth occur at primary crystallization [16, 29]. Crystallized macromolecules undergo further perfection in the second crystallization stage where the crystallization is slower as compared to primary stage.

The Avrami exponents $n$ were obtained from the slope of the primary crystallization stage where the crystallization rate is higher than the secondary crystallization stage. As can be seen from Table II, the Avrami exponent $n$ slightly decreased with increasing cooling rate. For primary crystallization, the values of the Avrami exponent $n$ of pure PLA was close to 2 , it shows that the crystallization mode of PLA is two-dimensional growth of nuclei $[21,30]$. The $n$ value of PLA in the presence of algae was found to vary between 1.70 to 2.60 at a given cooling rate, corresponding to various growth forms from rod-like to sphere-like [21, 31, 32]. Therefore, it can be concluded that the crystallization mode of PLA is partially changed by the incorporation of algae.

As shown in Table II, the crystallization rate constant $Z_{c}$ of pure PLA and PLA/green microalgae composites increased with increasing cooling rate. The $Z_{c}$ values of $\mathrm{PLA} /$ green microalgae composites were higher than that of pure PLA when compared at the same given cooling rate. This indicated that the incorporation of green microalgae enhanced the crystallization rate of PLA.

Another theory for the non-isothermal crystallization kinetics is the Ozawa model [33]. The non-linearity of the Avrami plots is often obtained, because the effect of cooling rate and the temperature variation by the time is not considered in the Avrami model. Ozawa modified the Avrami model by taking into consideration the cooling rate

$$
\ln (-\ln (1-X(t)))=\ln K(T)-m \ln \beta,
$$

where $K(T)$ represents the crystallization rate constant and $m$ is the Ozawa exponent. From the plots of $\ln (-\ln (1-X(t)))$ versus $\ln \beta$, the Ozawa exponent $m$ and the crystallization rate constant $K(T)$ can be obtained from the slope and intercept of the Ozawa plots, respectively. According to Eq. (6), the Ozawa plots of $\ln (-\ln (1-X(t)))$ versus $\ln \beta$ in the temperature range of $88-98^{\circ} \mathrm{C}$ for $\mathrm{PLA} /$ green microalgae composites are shown in Fig. 6. Linearity must be obtained as a restriction in order to apply the Ozawa model [34]. As can be seen from Fig. 6 that linearity criterion could not be satisfied in the Ozawa plots of PLA/green microalgae composites. There were two reasons for the failure of the Ozawa model to describe adequately the nonisothermal crystallization kinetics of PLA/green microalgae composites. The first one is the physical reason indicating deviation from linearity. The other one is the mathematical reason that the crystallization stage was different from each other at a given temperature and cooling rate. Moreover, it was concluded in the literature that the reasons of the invalidity are probably due to the strong secondary crystallization of PLA [35-37], and/or the dependence of lamellar thickness on crystallization temperature as well as heating function over the entire crystallization 

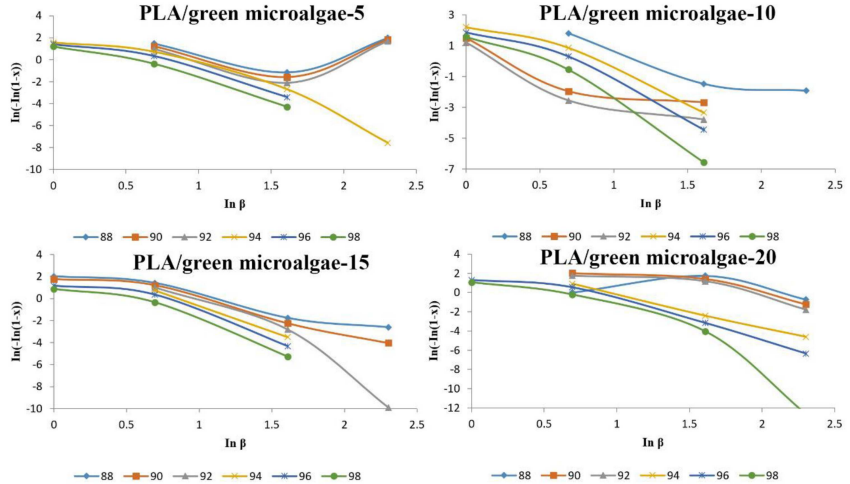

Fig. 6. Ozawa curves of PLA/green microalgae composites.

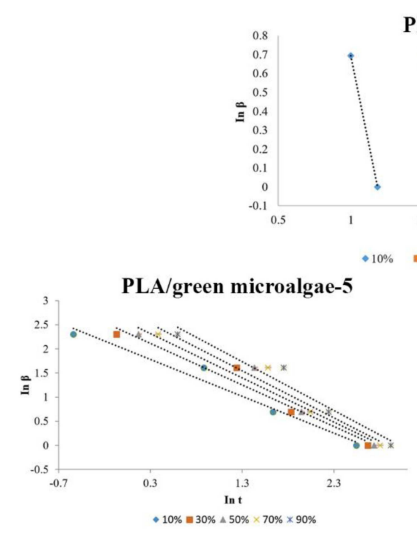

PLA

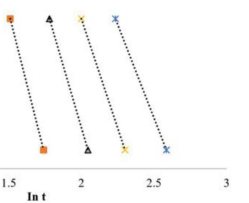

$30 \% \quad \Delta 50 \% \quad \times 70 \%$

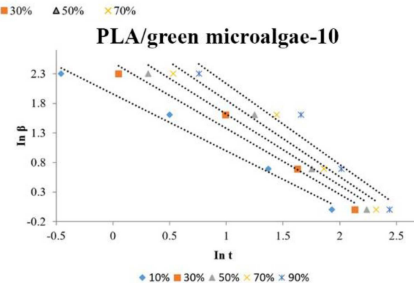

PLA/green microalgae-15

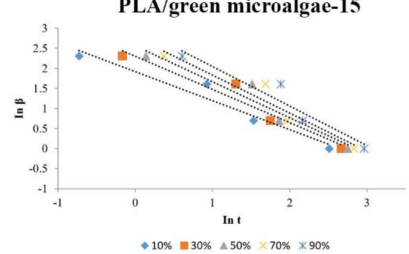

LA/green microalgae-20

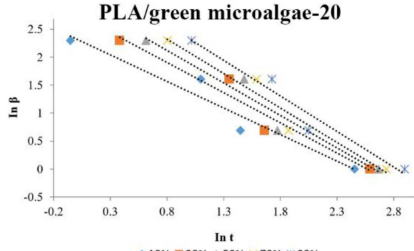

Fig. 7. Combined Avrami and Ozawa plots of PLA and PLA/green microalgae composites.

process [33, 35]. Therefore, the Ozawa model failed to describe the non-isothermal crystallization kinetics of PLA/green microalgae composites.

Mo et al. [38] proposed a different kinetic approach to describe the non-isothermal process more exactly by combining the Avrami and Ozawa equations based on the assumptions that at a given degree of crystallinity $X(t)$ is corrected to the cooling rate $\beta$ and crystallization time $t$ as follows:

$\ln Z+n \ln t=\ln K(T)-m \ln \beta$,

which can be further rewritten as follows:

$$
\ln \beta=\ln (F(T))-\alpha \ln t,
$$

where $\alpha=n / m$ is the ratio of the Avrami exponent $n$ to the Ozawa exponent $m$.
TABLE III

Combined Avrami and Ozawa parameters for PLA and PLA/green microalgae composites

\begin{tabular}{|c|c|c|c|}
\hline Sample & $X(t)[\%]$ & $\alpha$ & $F(T)$ \\
\hline \multirow{5}{*}{ PLA } & 10 & 3.75 & 4.45 \\
\hline & 30 & 3.02 & 5.27 \\
\hline & 50 & 2.61 & 5.35 \\
\hline & 70 & 2.31 & 5.31 \\
\hline & 90 & 1.97 & 5.10 \\
\hline \multirow{5}{*}{$\begin{array}{l}\text { PLA/green } \\
\text { microalgae-5 }\end{array}$} & 10 & 0.76 & 2.01 \\
\hline & 30 & 0.86 & 2.37 \\
\hline & 50 & 0.91 & 2.58 \\
\hline & 70 & 0.97 & 2.80 \\
\hline & 90 & 1.00 & 3.04 \\
\hline \multirow{5}{*}{$\begin{array}{l}\mathrm{PLA} / \text { green } \\
\text { microalgae-10 }\end{array}$} & 10 & 0.96 & 1.95 \\
\hline & 30 & 1.11 & 2.48 \\
\hline & 50 & 1.20 & 2.82 \\
\hline & 70 & 1.29 & 3.14 \\
\hline & 90 & 1.36 & 3.50 \\
\hline \multirow{5}{*}{$\begin{array}{l}\mathrm{PLA} / \text { green } \\
\text { microalgae-15 }\end{array}$} & 10 & 0.72 & 1.91 \\
\hline & 30 & 0.82 & 2.30 \\
\hline & 50 & 0.89 & 2.55 \\
\hline & 70 & 0.95 & 2.77 \\
\hline & 90 & 0.98 & 3.03 \\
\hline \multirow{5}{*}{$\begin{array}{l}\text { PLA/green } \\
\text { microalgae- } 20\end{array}$} & 10 & 0.95 & 2.32 \\
\hline & 30 & 1.07 & 2.76 \\
\hline & 50 & 1.16 & 3.05 \\
\hline & 70 & 1.23 & 3.31 \\
\hline & 90 & 1.27 & 3.59 \\
\hline
\end{tabular}

The kinetic parameter $F(T)=[K(T) / Z]^{1 / m}$ is the required cooling rate to reach a certain degree of crystallinity. $\quad F(T)$ has a definite and practical meaning for non-isothermal crystallization processes, which indicates the polymer crystallization rate [39]. Lower value of $F(T)$ means a higher crystallization rate under nonisothermal crystallization conditions [40]. The kinetic parameter $F(T)$ and the exponent of $\alpha$ can be determined by the intercept and the slope of the lines, respectively. According to Eq. (8), the combined Avrami-Ozawa plots of $\ln \beta$ versus $\ln t$ at a given relative crystallinity are shown in Fig. 7 and exhibit a good linear relationship, indicating that this kinetic approach was more effective in describing the non-isothermal crystallization kinetics of pure PLA and PLA/green microalgae composites.

The values of $F(T)$ and $\alpha$ at a given relative crystallinity are listed in Table III. As can be seen from Table III, the $\alpha$ values vary from 3.75 to 1.97 and from 0.72 to 1.36 for neat PLA and PLA/green microalgae composites, respectively, which indicates that the melt crystallization mode of PLA changed after the incorporation of green microalgae into PLA. The value of $F(T)$ gradually increased with increasing relative crystallinity for pure PLA and all composites. Moreover, the values of $F(T)$ of PLA decreased in the presence of green microalgae independent of green microalgae loading level at 


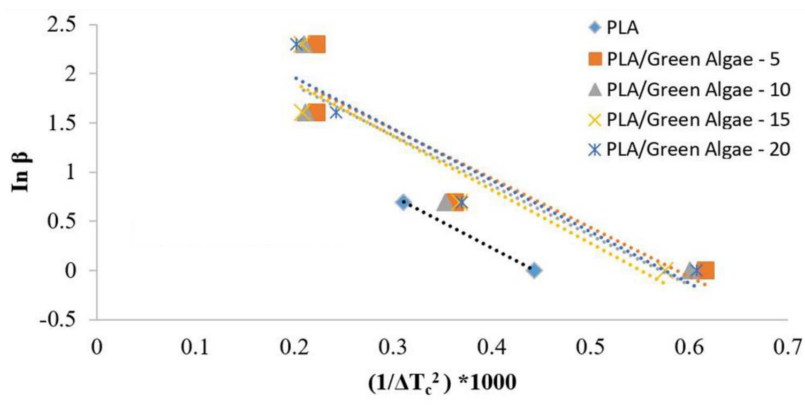

Fig. 8. Nucleating activity plots of PLA and PLA/green microalgae composites.

TABLE IV

Nucleating activities for PLA/green microalgae composites

\begin{tabular}{l|c|c|c}
\hline \hline \multicolumn{1}{c|}{ Sample } & $B$ & $B^{*}$ & $B^{*} / B$ \\
\hline PLA & 5.24 & - & - \\
PLA/green microalgae-5 & - & 2.72 & 0.52 \\
PLA/green microalgae-10 & - & 2.80 & 0.53 \\
PLA/green microalgae-15 & - & 2.91 & 0.55 \\
PLA/green microalgae-20 & - & 3.33 & 0.63
\end{tabular}

the same relative degree of crystallinity, that is, the melt crystallization rate of the PLA/ green microalgae composites was faster than that of pure PLA.

To determine the nucleating activity of the green microalgae for PLA, the Dobreva and Gutzow method were used in this study. In this model, a simple parameter called "nucleating activity" $\varphi$, was derived as follows in order to scale the effects of any filler as a nucleating agent [41]:

$$
\varphi=\frac{B^{*}}{B},
$$

where $B^{*}$ and $B$ are the parameters of heterogeneous and homogeneous medium, respectively. If the additive does not act as a nucleating agent, the value of $\varphi$ will be 1 , while for active additives, $\varphi$ approaches to 0 [42]. $B$ is defined as

$$
B=\frac{\omega \sigma^{3} V_{m}^{2}}{3 n k_{\mathrm{B}} T_{m}^{0} S_{m}^{2}},
$$

where $\omega$ is the geometric factor, $\sigma$ is the specific energy, $V_{m}$ is the molar volume of the crystallizing substance, $n$ is the Avrami exponent, $k_{\mathrm{B}}$ is the Boltzmann constant, $\Delta S_{m}$ is the melting entropy, and $T_{m}^{0}$ is the equilibrium melting temperature. Furthermore, the $B$ can be obtained from experimental values using the equation given below

$$
\ln \beta=C-\frac{B}{T_{c}^{2}},
$$

where $\beta$ is the cooling rate, $C$ is a constant, and $\Delta T_{c}$ is the supercooling $\left(T_{m}-T_{c}\right)$. Plots of the $\ln \beta$ versus $1 / \Delta T_{c}^{2}$ for the pure PLA and all composites are shown in Fig. 8. From the slope of the lines, the value of the $B$ and $B^{*}$ for pure PLA and PLA/green microalgae

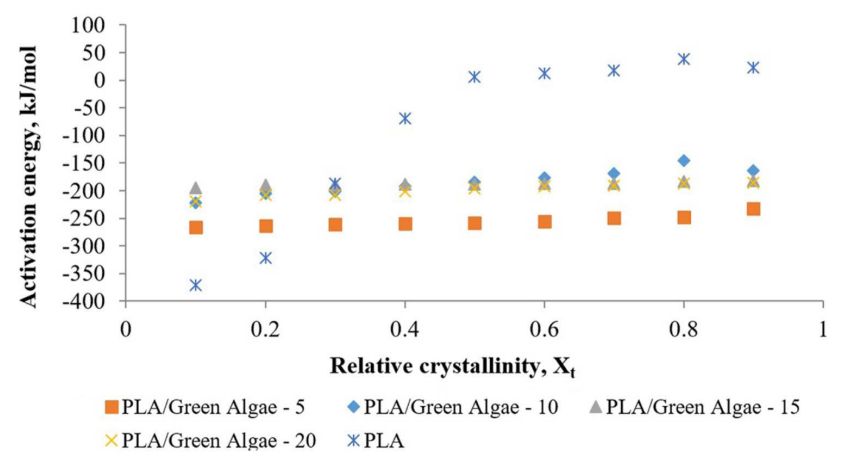

Fig. 9. Effective activation energy as a function of relative degree of crystallinity obtained during melt crystallization of neat PLA and PLA/green microalgae composites.

composites can be calculated, respectively. $B, B^{*}$, and $B^{*} / B$ are listed in Table IV. $B^{*} / B$ ratio for all PLA/green microalgae composites approached to zero, indicating that green microalgae was acting effectively as nucleating agent in PLA matrix.

In this study, activation energies of PLA and PLA/ green microalgae composites were calculated by using the Friedman method for each value of degree of crystallinity, ranging from 0.1 to 1 . The Friedman equation can be expressed as follows [43-45]:

$$
\ln \left(\frac{\mathrm{d} X}{\mathrm{~d} t}\right)_{X, t}=C-\frac{(\Delta E)_{X, t}}{(R T)_{X, t}}
$$

where $\mathrm{d} X_{t} / \mathrm{d} t$ is the instantaneous crystallization rate as a function of time for a given value of the relative crystallinity $\left(X_{t}\right), R$ is the universal gas constant, $\Delta E_{X, t}$ is the effective energy barrier of the process for a given value of $X_{t}$, and $C$ is a constant. By plotting the left hand side of Eq. (12) with respect to $1 / T_{X, t}$, a straight line must be obtained with a slope of $-\Delta E_{X} / R$. For this purpose, $\mathrm{d} X_{t} / \mathrm{d} t$ versus $1 / T_{X, t}$ were plotted for each cooling rate.

As can be seen from Fig. 9, the effective activation energy of PLA increased with increasing relative crystallinity. Activation energies at a given relative degree of crystallinity of PLA/green microalgae composites were significantly lower as compared to neat PLA, meaning a faster crystallization rate. Therefore, it can be concluded that addition of green microalgae to the PLA was in the favor of crystallization behavior of PLA. On the other hand, higher activation energies at low relative crystallinity values of composites as a comparison with PLA might be attributed to the agglomerations of green microalgae in PLA matrix.

\subsection{Polarized optical microscopy analysis}

The effect of the incorporation of green microalgae in PLA matrix as nucleating agent on the crystallization behavior was observed by polarized optical microscope (POM). Optical micrographs of non-isothermal crystallization process of PLA and 10 and $20 \mathrm{wt} \%$ green 


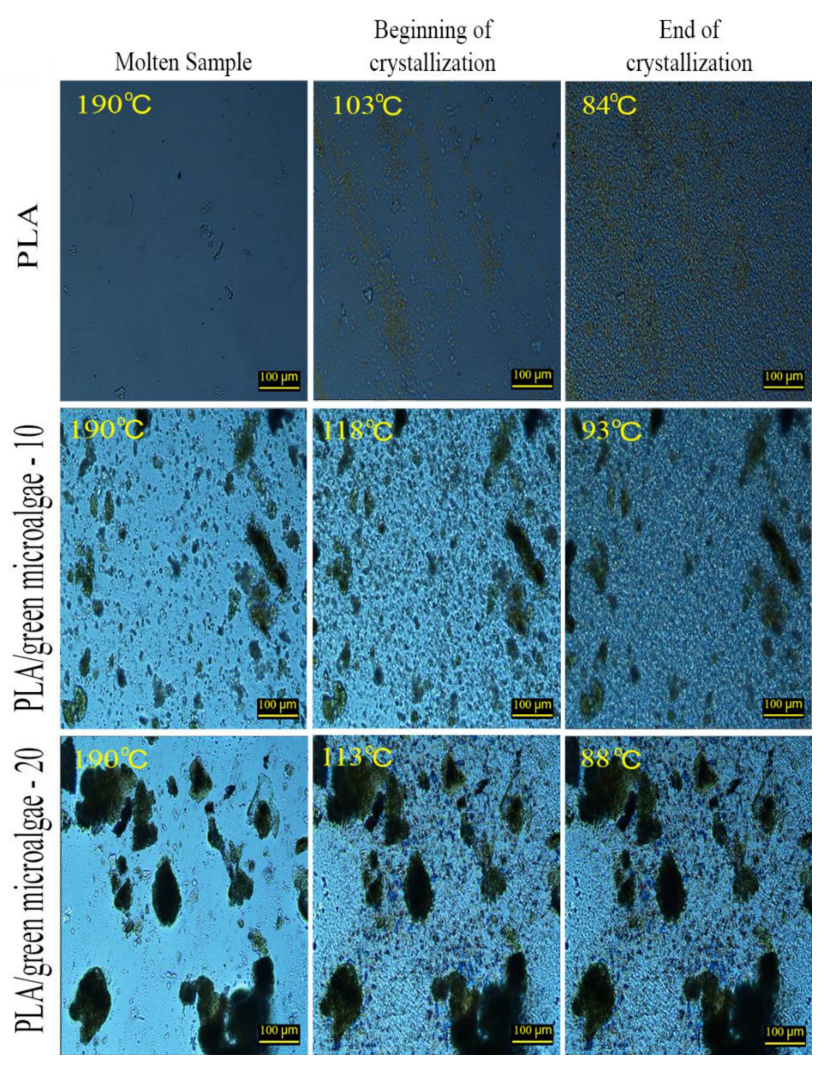

Fig. 10. Spherulite morphology of PLA and PLA/green microalgae composites.

microalgae filled PLA composites were obtained when the samples were cooled from $190^{\circ} \mathrm{C}$ to end of crystallization temperature at a cooling rate of $1^{\circ} \mathrm{C} / \mathrm{min}$ (Fig. 10). Pure PLA sample showed a clean uniform melt, while micro-scale aggregates of green microalgae were found in PLA matrix before crystallization. This further increased as the green microalgae content increased in the composites.

As can be seen from Fig. 10 that pure PLA began to crystallize around $103^{\circ} \mathrm{C}$ and crystallization process completed at $84^{\circ} \mathrm{C}$. After addition of green microalgae to the PLA, an increase in the overall crystallization rate of PLA obtained with an increase in the number of primary nucleation sites. The crystallization onset temperature shifted to significantly higher temperatures in the presence of green microalgae upon cooling. Moreover, nucleus density PLA was found to be higher in the presence of $10 \mathrm{wt} \%$ loading level of green microalgae. On the other hand, nucleus density decreased significantly with the addition of further content of green microalgae, due to the coarse dispersion of green microalgae in PLA matrix. It was found that, at lower loading levels of green microalgae, more crystals were able to nucleate and grow on the increased surface area of the interfaces. Similar results were reported in the literature that finely dispersed particles in polymer matrix were in the favor of crystallization rate [46].

\section{Conclusions}

The aim of this work was to investigate the effects of green microalgae on the non-isothermal crystallization kinetics of poly(lactic acid) (PLA). The filler content was 5, 10, 15, and $20 \%$ by weight. The nonisothermal crystallization kinetics and spherulitic morphology of PLA/green microalgae composites were investigated with DSC and POM. The results showed that green microalgae acted as a nucleating agent for PLA and crystallization onset temperature of PLA shifted higher upon cooling in the presence of green microalgaes. The Avrami based kinetic analysis pointed out that primary crystallization of samples was generally two-dimensional. The Ozawa method failed to describe the non-isothermal crystallization behavior of PLA/green microalgae composites. Combined Avrami and Ozawa model showed that the melt crystallization rate of the $\mathrm{PLA} /$ green microalgae composites was faster than that of pure PLA. Activation energies of nucleation for PLA decreased with the addition of green microalgae at higher relative degree of crystallinity. Green microalgae promoted the overall crystallization process of PLA matrix by improving the nucleation process. On the other hand, green microalgae at its higher loading levels retarded the rearrangement of PLA chain segments to some extent due to the agglomerations of green microalgaes in PLA matrix. The spherulitic morphology was observed with hotstage POM at non-isothermal conditions. Results showed that the nucleating density was higher in the presence of low content of green microalgae as compared to both pure PLA and PLA including $20 \mathrm{wt} \%$ green microalgae.

\section{Acknowledgments}

This work was granted by research foundation of Kocaeli University (Project No. 2017/048).

\section{References}

[1] L. Suryanegara, A.N. Nakagaito, H. Yano, Compos. Sci. Technol. 69, 1187 (2009).

[2] A.M. Harris, E.C. Lee, J. Appl. Polym. Sci. 107, 2246 (2008).

[3] R. Liao, B. Yang, W. Yu, C. Zhou, J. Appl. Polym. Sci. 104, 310 (2007).

[4] B. Suksut, C. Deeprasertkul, J. Polym. Environ. 19, 288 (2011).

[5] S. Fehri, P. Cinelli, M.B. Coltelli, I. Anguillesi, A. Lazzeri, Int. J. Chem. Eng. Appl. 7, 85 (2016).

[6] M.J. Carbone, M. Vanhalle, B. Goderis, P.V. Puyvelde, J. Polym. Eng. 35, 169 (2015).

[7] A. Shakoor, N.L. Thomas, Polym. Eng. Sci. 54, 64 (2014).

[8] M. Kodal, H. Sirin, G. Ozkoc, Polym. Compos. 38 1378 (2017).

[9] M. Kodal, H. Sirin, G. Ozkoc, Polym. Compos. 39, 2674 (2018). 
[10] M. Bulota, T. Budtova, Compos. Part A 73, 109 (2015).

[11] E. Chiellini, P. Cinelli, I. Ilieva, M. Martera, Biomacromolecules 9, 1007 (2008).

[12] E. Chiellini, P. Cinelli, I. Ilieva, F. Zimbardi, N. Kanellopoulos, B. Wilde, S. Verstichel, A. Pipino, B. Anders, J. Sassi, Int. J. Mater. Prod. Technol. 36, 47 (2009).

[13] S. Iannace, G. Nocilla, L. Nicolais, J. Appl. Polym. Sci. 73, 583 (1999).

[14] A. Barghini, V.I. Ivanova, S.H. Imam, E. Chiellini, J. Polym. Sci. A 48, 5282 (2010).

[15] E.M. Grima, E.H. Belarbi, F.G.A. Fernandez, A.R. Medina, Y. Chisti, Biotechnol. Adv. 20, 491 (2003).

[16] M. Ren, J. Song, Q. Zhao, Y. Li, Q. Chen, H. Zhang, Z. Mo, Polym. Int. 53, 1658 (2004).

[17] G.Z. Papageorgiou, D.S. Achilias, D.N. Bikiaris, G.P. Karayannidis, Thermochim. Acta 427, 117 (2005).

[18] S. Zhizhong, G. Weihong, L. Yongjun, L. Qiuying, W. Chifei, Polym. Bull. 62, 629 (2009).

[19] G.Z. Yang, X. Chen, W. Wang, M. Wang, T. Liu, C.Z. Li, J. Polym. Sci. B 45, 976 (2007).

[20] C. Zhang, B. Zhu, G. Ji, Y. Xu, J. Appl. Polym. Sci. 99, 2782 (2006)

[21] X.L. Jiang, S.J. Luo, K. Sun, X.D. Chen, Express Polym. Lett. 4, 245 (2007).

[22] A.G. Simanke, A.P. de Azeredo, C. de Lemos, R.S. Mauler, Polimeros 26, 152 (2016).

[23] M. Avrami, J. Chem. Phys. 7, 1103 (1939).

[24] M. Avrami, J. Chem. Phys. 8, 212 (1940).

[25] A.R. Adhikari, K. Lozano, M. Chipara, J. Compos. Mater. 46, 823 (2011)

[26] X. Yang, Y. Wu, K. Wei, Materials 11, 2094 (2018).

[27] A. Jeziorny, Polymer 19, 1142 (1978).

[28] X. Yong, S. Songmin, H. Jian, W. Sinchi, J. Mater. Sci. 46, 4085 (2011).
[29] S. Gumus, G. Ozkoc, A. Aytac, J. Appl. Polym. Sci. 123, 2837 (2012).

[30] D. Garcia, J. Polym. Sci. Polym. Phys. E 22, 2063 (1984).

[31] P. Sajkiewicz, L. Carpaneto, A. Wasiak, Polymer 42, 5365 (2001).

[32] S.A. Jabarin, J. Appl. Polym. Sci. 34, 85 (1987).

[33] T. Ozawa, Polymer 12, 150 (1971).

[34] X. Li, W. Guo, Q. Zhou, S. Xu, C. Wu, Polym. Bull. 59, 685 (2007).

[35] N. Shi, Q. Dou, J. Therm. Anal. Calorim. 119, 635 (2015).

[36] J. Cho, S. Baratiana, J. Kim, F. Yeh, B.S. Hsiao, J. Runt, Polymer 44, 711 (2003).

[37] S.C. Schmidt, M.A. Hillmyer, J. Polym. Sci. B 39, 300 (2001).

[38] T. Liu, Z. Mo, S. Wang, H. Zhang, Polym. Eng. Sci. 37, 568 (1997)

[39] C. Ge, L. Shi, H. Yang, S. Tang, Polym. Compos. 31 , 1504 (2010)

[40] J.W. Huang, Y.C. Hung, Y.L. Wen, C.C. Kang, M.Y. Yeh, J. Appl. Polym. Sci. 112, 3149 (2009).

[41] A. Dobreva, I. Gutzow, J. Non-Cryst. Solids 162, 13 (1993).

[42] G.Z. Papageorgiou, D.S. Achilias, S. Nanaki, T. Beslikas, D. Bikiaris, Thermochim. Acta 511, 129 (2010).

[43] J.W. Huang, Y.L. Wen, C.C. Kang, M.Y. Yeh, S.B. Wen, Thermochim. Acta 465, 48 (2007).

[44] J.N. Martins, O. Bianchi, C.H. Wanke, C.D. Castel, J. Polym. Res. 22, 224 (2015).

[45] W.S. Saeed, A.B. Al-Odayni, A.A. Alghamdi, A. Alrahlah, T. Aouak, Crystals 8, 452 (2018).

[46] A. Pei, Q. Zhou, L.A. Berglund, Compos. Sci. Technol. 70, 815 (2010). 